Western University

Scholarship@Western

Medical Biophysics Publications

Medical Biophysics Department

$1-2015$

Unified voxel- and tensor-based morphometry (UVTBM) using registration confidence.

Ali R Khan

Western University, alik@robarts.ca

Lei Wang

Mirza Faisal Beg

Follow this and additional works at: https://ir.lib.uwo.ca/biophysicspub

Part of the Medical Biophysics Commons

Citation of this paper:

Khan, Ali R; Wang, Lei; and Beg, Mirza Faisal, "Unified voxel- and tensor-based morphometry (UVTBM) using registration confidence." (2015). Medical Biophysics Publications. 39.

https://ir.lib.uwo.ca/biophysicspub/39 


\title{
Unified voxel- and tensor-based morphometry (UVTBM) using registration confidence
}

\author{
Ali R. Khan ${ }^{\mathrm{a}}$, Lei Wang ${ }^{\mathrm{b}}$, Mirza Faisal Beg ${ }^{\mathrm{c}, *}$ \\ ${ }^{a}$ Department of Medical Biophysics, Robarts Research Institute, Western University, London, Ontario, Canada \\ ${ }^{\mathrm{b}}$ Department of Psychiatry and Behavioral Sciences and Radiology, Northwestern University, Feinberg School of Medicine, Chicago, IL, USA \\ ${ }^{\text {c }}$ School of Engineering Science, Simon Fraser University, Burnaby, British Columbia, Canada
}

\section{A R T I C L E I N F O}

\section{Article history:}

Received 6 May 2013

Received in revised form 16 April 2014

Accepted 24 April 2014

Available online 30 August 2014

\section{Keywords:}

MRI

Brain registration

Morphometry

Diffeomorphisms

Atlases

Voxel-based morphometry

Tensor-based morphometry

\begin{abstract}
A B S T R A C T
Voxel-based morphometry (VBM) and tensor-based morphometry (TBM) both rely on spatial normalization to a template and yet have different requirements for the level of registration accuracy. VBM requires only global alignment of brain structures, with limited degrees of freedom in transformation, whereas TBM performs best when the registration is highly deformable and can achieve higher registration accuracy. In addition, the registration accuracy varies over the whole brain, with higher accuracy typically observed in subcortical areas and lower accuracy seen in cortical areas. Hence, even the determinant of Jacobian of registration maps is spatially varying in their accuracy, and combining these with VBM by direct multiplication introduces errors in VBM maps where the registration is inaccurate. We propose a unified approach to combining these 2 morphometry methods that is motivated by these differing requirements for registration and our interest in harnessing the advantages of both. Our novel method uses local estimates of registration confidence to determine how to weight the influence of VBM- and TBM-like approaches. Results are shown on healthy and mild Alzheimer's subjects $(N=150)$ investigating age and group differences, and potential of differential diagnosis is shown on a set of Alzheimer's disease $(N=34)$ and frontotemporal dementia $(N=30)$ patients compared against controls $(N=14)$. These show that the group differences detected by our proposed approach are more descriptive than those detected from VBM, Jacobian-modulated VBM, and TBM separately, hence leveraging the advantages of both approaches in a unified framework.
\end{abstract}

(c) 2015 Elsevier Inc. All rights reserved.

\section{Introduction}

Voxel-based morphometry (VBM) (Ashburner and Friston, 2000; Mechelli et al., 2005) is a widely used whole-brain analysis method for characterizing morphologic shape differences using brain magnetic resonance imaging (MRI) and has been useful for investigating a wide range of neurological and psychiatric diseases, tracking normal development of the brain, and assessing the structural effect of neuroplasticity in the human brain. In essence, VBM is a voxelwise comparison of tissue segmentation maps that have been brought into global alignment (through spatial normalization) and smoothed with an isotropic Gaussian kernel. To deal with brain regions that may expand or contract through the spatial normalization process, a local measure of contraction or expansion is often

\footnotetext{
LW and MFB have contributed equally to this work.

* Corresponding author at: School of Engineering Science, Simon Fraser University, Burnaby, British Columbia, Canada V5A 1S6. Tel.: +1 778782 5696; fax: +1 778 7824951.

E-mail address: mfbeg@sfu.ca (M.F. Beg).
}

used to modulate the density maps. This procedure, known as Jacobian modulation, uses the determinant of the Jacobian derived from the transformations that register each brain to the common space (Davatzikos et al., 2001), thus dealing with gross-volume differences that may exist and leading to a more natural interpretation of tissue volume with respect to the unwarped maps. This spatial normalization commonly consists of an initial 12-parameter affine registration followed by a nonlinear registration step. Because the process of spatial normalization in VBM is meant to only globally align the brain, the registration is thus limited in the degrees of freedom (dof) used to transform the images. Previous work has used transformations modeled with a linear combination of smooth basis functions, as in Ashburner and Friston (2000), or those spline parameterizations with wide ( $>10 \mathrm{~mm}$ ) control-point spacing, as in Douaud et al. (2007). Limiting the dof is essential to VBM without Jacobian modulation, because if the dof were high enough to allow for complete alignment of the anatomy, the differences would not be detected in the gray-matter (GM) density maps (Thacker, 2005). Note that depending on the type of anatomy being registered, complete alignment may or may not be achievable. For example, 
many regions in the cerebral cortex have individualized folding patterns that would confound complete alignment even with high dof, whereas many subcortical nuclei have simpler morphology and could be completely aligned given enough dof.

A recent large-scale evaluation of brain registration carried out by Klein et al. (2009) showed that methods with highly deformable transformation models (high dof) perform significantly more accurate registration than those with limited dof. In particular, diffeomorphic registration algorithms that model the transformation as a smoothly varying fluid flow while ensuring smoothness and invertibility (Avants et al., 2008; Beg et al., 2005; Khan et al., 2013) have been shown to outperform competing methods in many situations. These methods in principle cannot be directly used with VBM because of the previous considerations; thus, morphometries based on these high dof registration techniques are deformation or tensor based, that is, statistics are performed on the deformation fields (deformation-based morphometry, Ashburner et al., 1998), or the Jacobian tensors of these deformation fields (tensor-based morphometry [TBM], Davatzikos et al., 1996), and do not make use of the smoothed tissue segmentations. However, these techniques rely on a high degree of registration accuracy and may be underpowered in regions where registration accuracy is lower.

It is known that issues such as the size, shape, and folding pattern of structures or local image contrast changes can effect the accuracy; the cortical regions of the brain pose significant challenges for even the most advanced brain registration algorithms (Klein et al., 2009), and, thus, uncertainty is typically higher in these regions. Furthermore, in the case of disease-specific analysis, the particular disease can potentially have very different spatial atrophy profiles and, therefore, also influence the uncertainty in registration as a function of location in the brain. In regions where we have more uncertainty in registration, the Jacobian maps would contain more noise, and, thus, a VBM approach would be more sensitive and powerful in these regions. In regions where we have less uncertainty in registration, the Jacobian contains more information, and, thus, a TBM approach would be preferred in these regions. Note that VBM and TBM have similar interpretations as the proportion of tissue volume in a subject over an atlas, and both rely on the same image features (tissue contrast from T1-weighted structural images) to assess the same biological phenomenon (volume change). Because of this inherent similarity, they are ideal candidates for a unified method that combines the advantages of each approach into a single feature.

We propose here a novel unification of VBM and TBM that uses local estimates of registration confidence or inversely, uncertainty, to select whether VBM, TBM, or a combination should be used at a given voxel. To perform this unification, we observe that effectively, in regions where we have high confidence in registration, the determinant of Jacobian (TBM) is used, in regions where we have lower confidence in registration, the tissue segmentation map (VBM) is used, and in regions with an intermediate level of registration confidence, a smooth transition between the 2 methods is used. Given a group-wise registration setting, we show how the registration confidence can be estimated locally at each voxel and how to use that to unify the TBM and VBM approaches in 1 single statistical parametric map (SPM). The advantage of our locally adaptive registration-confidence weighted approach is that the choice of VBM or TBM or a combination occurs at each voxel based on the registration confidence. In this way, we can produce a single SPM quantifying brain morphometry based on the principles of both VBM and TBM. In addition, the registration accuracy or confidence map is also useful in interpreting desired results in the context of group-wise registration accuracy over the whole brain.

\section{Methods}

We will first describe our general framework for estimation of registration confidence and unification of VBM and TBM based on local measures of confidence in aligning tissue segmentations. Then, we will describe our implementation of this framework that involves a multistructure group-wise registration to an average template. We applied our unified morphometry framework to an open access set of 150 elderly subjects, healthy and Alzheimer's disease $(A D)$, and examined the effect of age and group, showing the results for various parameter choices. We also applied it to a database of frontotemporal dementia (FTD) and AD patients to investigate the use of whole-brain morphometry in differential diagnosis of neurodegenerative disease patients.

\subsection{Estimating registration confidence}

In VBM and TBM, brain MRI is registered to a common template, where analysis of tissue density and deformation derivatives can take place. For a given set of subjects, we would like to estimate, at a spatially local level, the uncertainty in aligning the anatomy, to provide an estimate of confidence. Estimating spatial uncertainty in registration is an open problem, and there has been interest in recent years to better inform users in clinical applications of registration. Hub et al. (2009) measured uncertainty by examining how random perturbations of the final registration transform affect a given cost function. Kybic (2010) used bootstrap resampling to estimate the uncertainty of the covariance matrix and evaluated their approach on the simple case where transformations are restricted to 2-dimensional translations. More generally, spatial uncertainty in pairwise registrations can be modeled in a Bayesian framework as the variance in the posterior distribution, because the posterior is defined as the distribution of deformation parameters. Risholm et al. (2013) used computationally intensive Markov Chain Monte Carlo methods to sample the posterior distribution and estimate the uncertainty as the variance of parameters in this distribution, and Simpson et al. (2012) proposed a more efficient variational Bayes model for the estimation of uncertainty.

For the application to group studies, we are interested in the uncertainty of group-wise registration, which is dependent both on the pairwise spatial uncertainty, as explored in the previous studies, and the anatomic variability across the subjects. For the sake of extensibility to arbitrary registration methods, we would like to use an approach of measuring uncertainty that is not computationally expensive (Risholm et al., 2013) or require a custom formulation and optimization of the registration (Simpson et al., 2012). Thus, instead of specific estimation of the posterior probability distribution, we represent the registration confidence as the residual spatial variability remaining after group-wise registration. To define this, we first consider the general situation where corresponding entities exist across MR images, which could be various representations of anatomy including landmarks, curves, volumes, or surfaces. Then, the registration confidence at each entity, $r$, can be defined as the probability that corresponding entities, $r_{i}$, from all subjects, $i=1, \ldots, N$, are within $\varepsilon$ distance of the mean location of that entity, $\mu_{r}$ :

$p_{\mathrm{c}}=p\left(\left\|r_{i}-\mu_{r}\right\|<\varepsilon\right)$

Applying Chebyshev inequality gives us a lower bound of the confidence as:

$p_{\mathrm{c}} \geq 1-\frac{\sigma_{r}^{2}}{\varepsilon^{2}}$ 
where $\sigma_{r}^{2}$ is the spatial variance at entity $r$. With this formulation, the minimum confidence at a given location becomes a simple computation involving the variance of each landmark and the parameter $\varepsilon$. The free parameter $\varepsilon$ specifies the tolerance or margin for registration uncertainty; increasing the tolerance leads to effectively higher confidence, $p_{c}$, and decreasing the tolerance leads to effectively lower confidence.

In this article, we use GM tissue segmentations as the MRI entities, using the nearest distance between GM tissue segmentations, $S$, to approximate Equation (1):

$\left\|r_{i}-\mu_{r}\right\| \approx \operatorname{Dist}\left(S_{i}(x), \bar{S}(x)\right)$

$\left\|r_{i}-\mu_{r}\right\|=\left\{\begin{array}{cc}0 & \text { if } S_{i}(x)=\bar{S}(x), \\ \left(\left|D T_{S_{i}}(x)\right|+\left|D T_{\bar{S}}(x)\right|\right) & \text { if } S_{i}(x) \neq \bar{S}(x),\end{array}\right.$

where DT denotes the distance transform of a binary image, $S_{i}(x)$ is a tissue segmentation for subject $i$ at spatial location $x$, and $\bar{S}$ is a consensus tissue segmentation (e.g., averaged and thresholded) based on all subjects. Equation (4) provides a measure of how close or far the nearest boundaries are for every voxel where the segmentations do not agree. Given a number of images that have been registered into a common space, and GM tissue segmentations, we can apply this approximation to efficiently compute $\sigma^{2}$ in Equation (2) for each spatial location and thus generate a registration confidence map based on a specific setting of $\varepsilon$.

\subsection{Unified voxel- and tensor-based morphometry}

Once we have an estimate of the registration confidence at a given spatial location in the template, we can use this to determine whether a VBM- or TBM-like approach is suitable. Recall that in traditional VBM, statistics are performed on the tissue segmentation probabilities (or densities) of each subject transformed to the template, referred to here as $S$. In TBM, statistics are performed on the determinant Jacobian of the transformations, $|D \phi|$. In a unified model, if registration confidence is very low, then we should place less weight on the determinant Jacobian, because this is derived directly from the deformation fields from registration. Conversely, if the registration confidence is very high, then we should place less weight on the density maps, because the anatomy is expected to be well aligned in this case. This leads us to the following simple model for the unified feature, $U$, at spatial location, $x$ :

$U(x)=S(x)^{1-p_{c}(x)} \cdot|D \phi(x)|^{p_{c}(x)}$,

where the influence of the density map, $S$, in the template space, and determinant Jacobian, $|D \phi|$, are weighted by the registration confidence, $p_{c}$. If $p_{c}=0$, then we have the traditional VBM approach, and if $p_{\mathrm{c}}=1$, we have the traditional TBM approach. At the midpoint, $p_{\mathrm{c}}=5$, we have $U(x)=\sqrt{S(x) D \phi(x)}$, an approach that is proportional to Jacobian-modulated VBM or mass-preserving maps, where we effectively have volume in place of density. We used this multiplicative model for combining the 2 approaches, because it directly relates to the VBM without modulation, VBM with modulation, and TBM approaches.

\subsection{Implementation}

We now describe our specific implementation of unified voxel- and tensor-based morphometry (UVTBM), namely, how we generated tissue segmentations, performed the registration, and estimated the registration confidence map. Note that the UVTBM model described earlier can be applied with typically used segmentation and registration techniques as long as the transformation model used in the registration is highly deformable.

\subsubsection{Segmentation and registration}

For segmentation and MRI preprocessing, we used the FreeSurfer image analysis suite (Fischl et al., 2002, 2004). Segmentations of the subcortical structures were obtained from FreeSurfer's subcortical processing stream (Fischl et al., 2002), and the cortical GM structures were obtained from a volumetric mask of the cortical surfaces (Fischl et al., 2004). These were both combined to form a single GM segmentation.

We used a group-wise multistructure registration approach (Khan et al., 2013), which used subcortical segmentations and cortical parcellations (Fischl et al., 2004) along with the MRI to concurrently drive a diffeomorphic registration (Christensen et al., 1996). The Christensen registration algorithm was employed instead of large deformation diffeomorphic metric mapping because of its efficiency, especially when employed in a multistructure group-wise approach. The FreeSurfer image analysis suite (Dale et al., 1999; Fischl et al., 1999, 2002) was used to generate subcortical segmentations and cortical parcellations making up the multistructure image set. We used the following 8 subcortical structures, left and right inclusive: lateral ventricles, caudate nucleus, putamen, pallidum, nucleus accumbens, thalamus, hippocampus, and amygdala. For cortical structures, we used the following 33 labels, left and right inclusive: bankssts (banks around the superior temporal sulcus), caudal anterior cingulate, caudal middle frontal, corpus callosum, cuneus, entorhinal, fusiform, inferior parietal, inferior temporal, isthmus cingulate, lateral occipital, lateral orbitofrontal, lingual, medial orbitofrontal, middle temporal, parahippocampal, paracentral, pars opercularis, pars orbitalis, pars triangularis, pericalcarine, postcentral, posterior cingulate, precentral, rostral anterior cingulate, rostral middle frontal, superior frontal, superior parietal, superior temporal, supramarginal, frontal pole, temporal pole, and transverse temporal. Including the MRI as the first structure, we have 43 structure channels for each hemisphere making up our multistructure set. To limit computational and memory requirements, we construct a bounding box around the template brain and use this subvolume in registration instead of the entire $256 \times 256 \times 256$ volume. The bounding box is defined using the extents of the skull-stripped brain, allowing for at least 16 voxels (16 $\mathrm{mm}$ ) of padding at each boundary, and also ensuring the dimensions of the image are divisible by 16 , such that this level of data parallelization could be used. The bounding box is applied to both the MRI and multistructure set of the template. The groupwise approach was initialized by first selecting a single subject as an exemplar template, affinely registering all subjects to it, and then averaging the registered images. For the diffeomorphic registration, a multiscale smoothing scheme was used that successively decreased the viscosity parameter, $\alpha$, after each iteration. The sequence used was $\alpha=2,1,0.5,0.1$, with a fixed $\gamma=0.01$, which was chosen based on experiments quantifying effective smoothing to be equivalent to $20-, 15-, 10-$, and $5-\mathrm{mm}$ effective smoothing, respectively, as shown in Appendix B of Khan (2011).

Group-wise registration was carried out on the $N$ T1 images in the database, $I_{i}$, generating transformations for each subject, $\phi_{i}$, that comprised both the affine and diffeomorphic transformations to the template. GM segmentation maps, $S_{i}$, were then transformed to the template, $S_{i}^{0}=S_{i} \phi^{-1}$, and the determinant Jacobians were also computed for each subject, $|D \phi|$. 


\subsubsection{Unified VTBM}

Once the registration confidence map, $p_{\mathrm{c}}$, was computed for a given $\varepsilon$ in the template space, we applied Equation (5) to generate unified density and Jacobian maps, $U_{i}$, for each subject:

$U_{i}(x)=S_{i}(x)^{1-p_{c}(x)} \cdot\left|D \phi_{i}(x)\right|^{p_{c}(x)}$

The normalized FreeSurfer GM segmentations for each subject and the registration confidence map were smoothed by a Gaussian filter (10-mm full-width half maximum) before applying Equation (5). The determinant Jacobian maps were not smoothed because the diffeomorphic registration already ensured a high level of regularity in the maps. We performed statistical analysis using the SurfStat package (http://www.math.mcgill.ca/keith/surfstat/) to generate SPMs using the UVTBM maps, using random field theory correction based on peak $T$ statistics and cluster extent.

To compare against traditional VBM, Jacobian-modulated VBM, and TBM approaches, we used UVTBM with confidence maps, $p_{c}$, set to a globally constant value. By setting $p_{\mathrm{c}}=0$, we have pure VBM, setting $p_{\mathrm{c}}=0.5$, we have Jacobian-modulated VBM, and setting $p_{\mathrm{c}}=$ 1 , we have a pure TBM. We compared against these approaches in this way to ensure that identical preprocessing steps and registrations are used and, thus, do not confound the comparison, as would occur if a different software package (SPM and FSL) were used instead. Note that the setting of $p_{\mathrm{c}}=0.5$ does not exactly relate to the Jacobian-modulated VBM as employed in the aforementioned software packages, as we do not smooth the Jacobian maps here. Because Jacobians are not smoothed in TBM implementations, we also chose not to smooth them here so the extremes of zero registration confidence and full registration confidence relate to pure VBM and TBM, respectively.

\section{Experiments and results}

\subsection{Alzheimer's disease}

We used MRI datasets from the Open Access Series of Imaging Studies (http://www.oasis-brains.org), referred to as database 1, to evaluate morphometry in an elderly population. We used baseline scans from a dataset consisting of 150 subjects, aged 60-96 years, with 64 subjects characterized with very mild to mild Alzheimer's disease (Marcus et al., 2010). This database was chosen to facilitate comparison with previous related studies on the same database (Khan, 2011). We first performed group-wise registration on all 150 subjects, and Fig. 1 shows representative axial slices of the average MRI and GM segmentations for the 150 subjects. Then, we generated the registration confidence maps for varying $\varepsilon$, shown in Fig. 2, where one can see high confidence in the subcortex and temporal cortex and lower confidence in the posterior cortex.

We used a general linear model $M=1+$ age + gender + group and performed analyses for the main effect of age and group (nondemented vs. demented) and used random field theory multiple comparison correction to find significant voxels and clusters. Figure 3 shows statistical maps with main effect for age comparing our UVTBM approach (with a range of error tolerances, $\varepsilon$ ) with traditional approaches of VBM (setting $p_{\mathrm{c}}=0$ ), Jacobian-modulated $\operatorname{VBM}\left(p_{\mathrm{c}}=0.5\right)$, and TBM $\left(p_{\mathrm{c}}=1\right)$. Here, we see that VBM and TBM reveal complementary differences in the cortical regions, with VBM more sensitive posteriorly (where registration confidence is lower) and TBM anteriorly (where registration confidence is higher); yet, our UVTBM approach is sensitive to differences revealed by both. Figure 3 also illustrates the dependency on the uncertainty tolerance $(\varepsilon)$, as it affects whether a predominant VBM or TBM approach is to be used in the cortex; we see that UVTBM with high tolerance $(\varepsilon=0.75)$ results in higher overall confidence in the cortex and a map similar to Jacobian-modulated VBM. Figure 4 shows the statistical maps with main effect for group, effectively showing where demented subjects have significantly decreased GM density, volume, and increased contraction. We see much of the differences in the medial temporal lobe, as is expected for dementia of Alzheimer's type. In this comparison, the results for UVTBM are very similar to that of modulated VBM and TBM, indicating that much of the information here is encoded in the determinant Jacobian. Figure 4 also reveals that the range of uncertainty tolerance $(\varepsilon)$ evaluated here did not have a major effect on the resulting maps; however, a larger range of values would reveal behavior similar to VBM or TBM.

\subsection{Potential for differential diagnosis}

Differential diagnosis of dementia is often difficult because patients may exhibit the same cognitive impairments with different neuropathology. To investigate the potential of our UVTBM approach to discriminate between various diseases, we used data
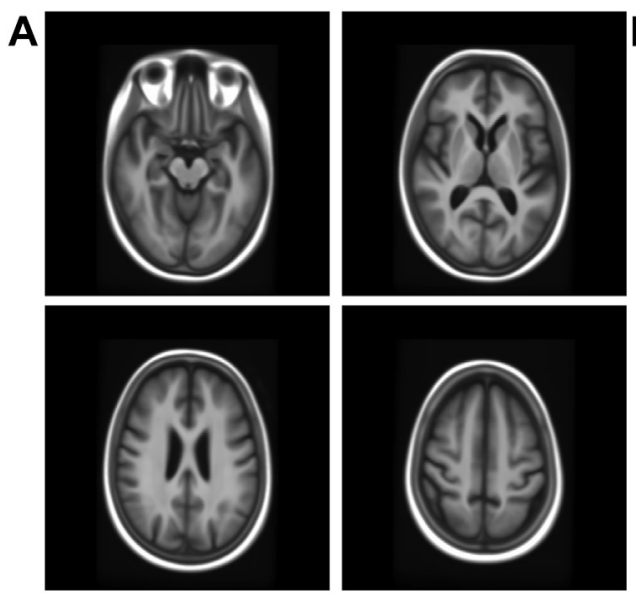

Average T1 MRI
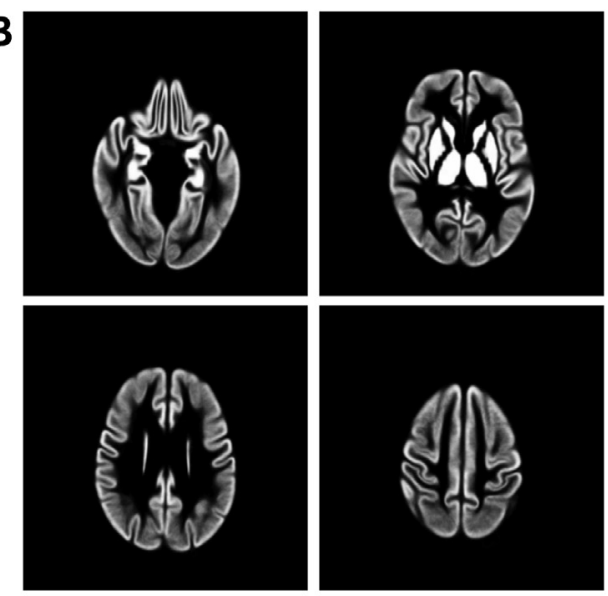

Average gray matter

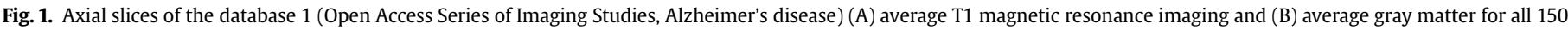

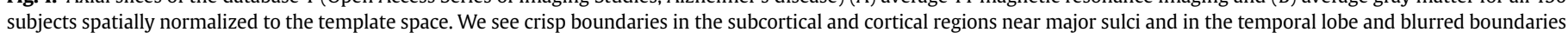
in many other cortical regions indicating the level of registration accuracy. 


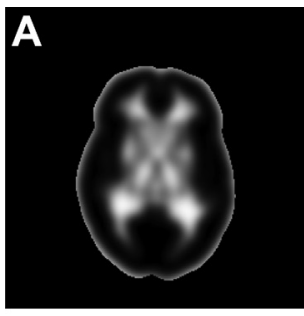

$P_{c}, \epsilon=0.25$

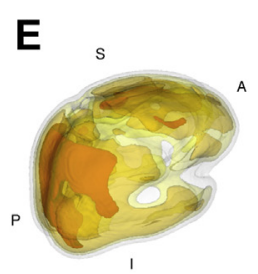

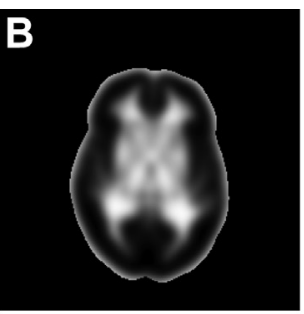

$P_{c}, \epsilon=0.5$

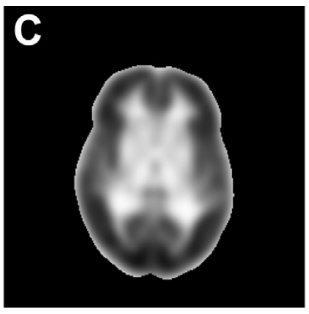

$P_{c}, \epsilon=0.75$

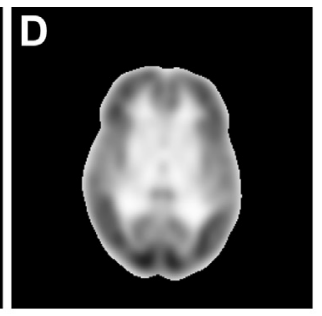

$P_{c}, \epsilon=1.0$

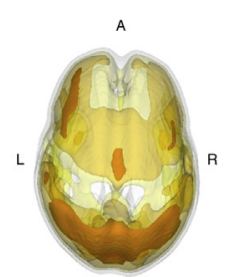

$P_{c}, \epsilon=0.5$

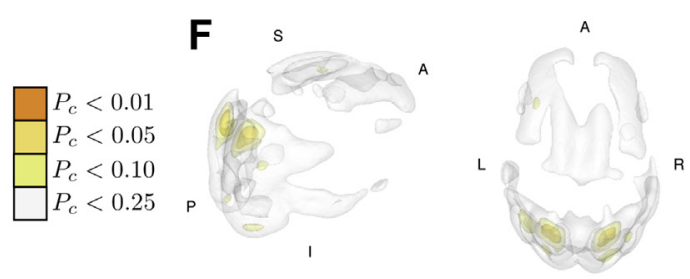

$P_{c}, \epsilon=0.75$

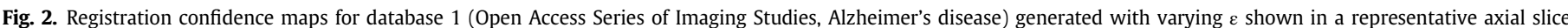

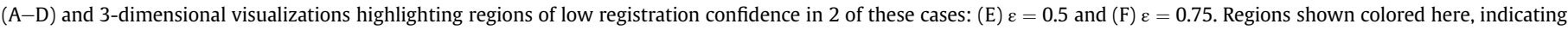

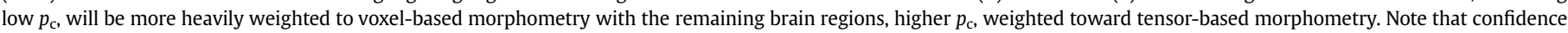

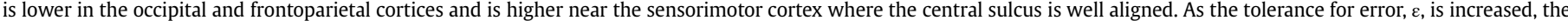

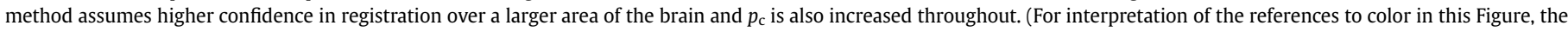
reader is referred to the web version of this article.)

from a study consisting of 14 healthy control subjects (aged 49-62 years), 34 probable AD patients (48-62 years), and 30 FTD patients (48-62 years) with magnetization-prepared rapid gradient-echo scans acquired on a 1.5-T scanner, referred to as database 2 .

We performed group-wise registration using all subjects in the combined database, applied our VTBM approach with varying $\varepsilon$, and compared this against VBM and TBM approaches. We compared each group with the control group to observe how the patterns of neurodegeneration differ in each disease by computing statistical parametric maps with the general linear model, controlling for age and gender, $M=1+$ group + age + gender, where the group was modeled with separate dummy variables for each of the 3 groups. Figure 5 shows the $T$ statistics and significant maps comparing the control group with each other group. We see that these findings agree very well with the previous known neuropathology and atrophy patterns of these diseases. In the probable Alzheimer's group, we see cortical atrophy that is stronger in posterior regions of the brain and expansion of the body of the lateral ventricles. The FTD group exhibits a contrasting pattern, with cortical atrophy mainly restricted to the frontal and temporal lobes and with expansion seen in the anterior horns of the lateral ventricles, hallmark patterns of differences between these 2 dementias
A
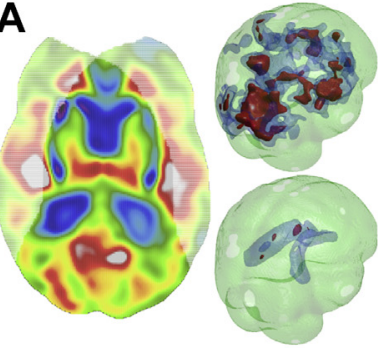

UVTBM, $\epsilon=0.25$

D
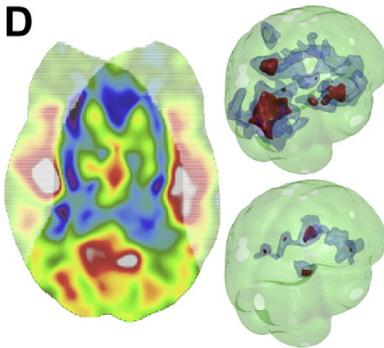

VBM, $P_{c}=0$
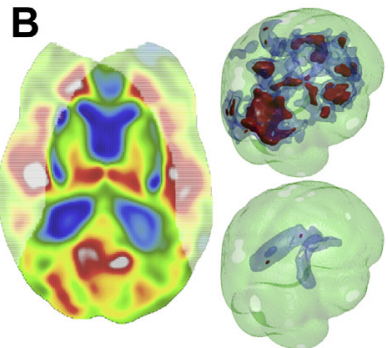

UVTBM, $\epsilon=0.5$

E

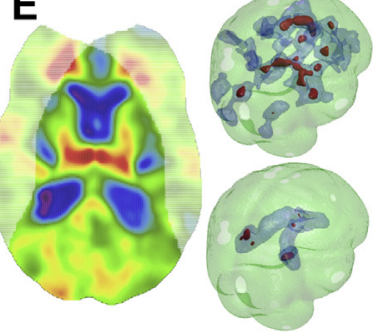

Jac. Mod. VBM, $P_{c}=0.5$
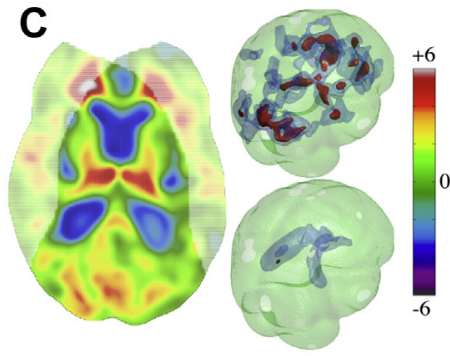

UVTBM, $\epsilon=0.75$

$\mathbf{F}$

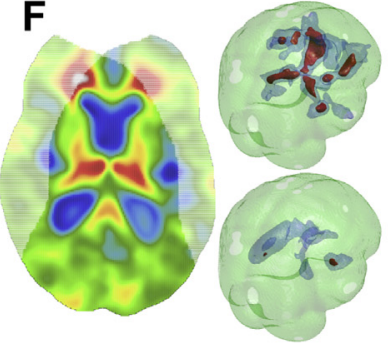

TBM, $P_{c}=1$

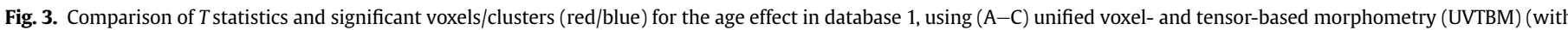

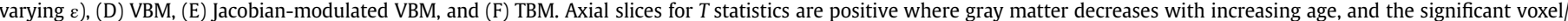

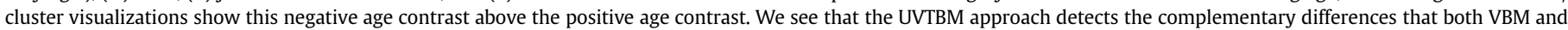
TBM detect. (For interpretation of the references to color in this Figure, the reader is referred to the web version of this article.) 


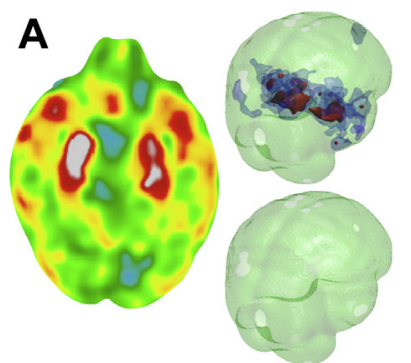

UVTBM, $\epsilon=0.25$

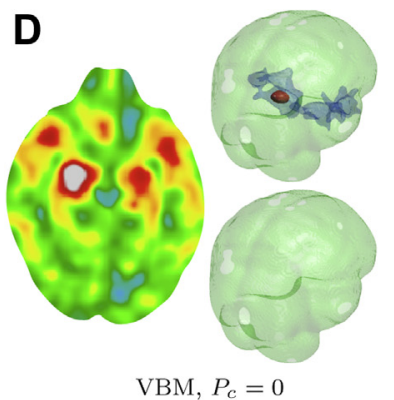

B

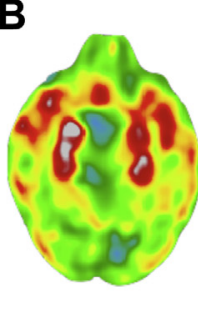

UVTBM, $\epsilon=0.5$

E

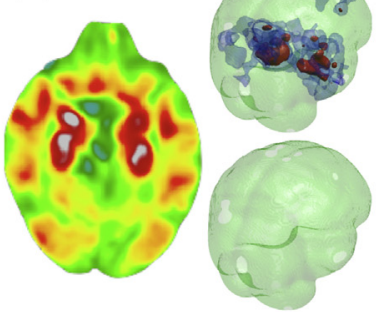

Jac. Mod. VBM, $P_{c}=0.5$

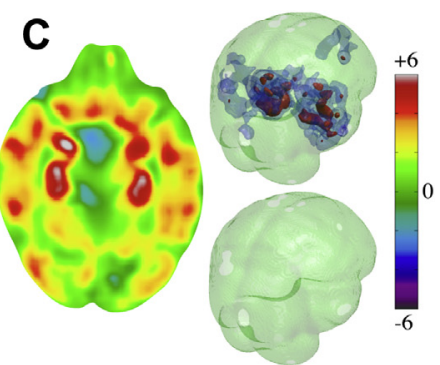

UVTBM, $\epsilon=0.75$

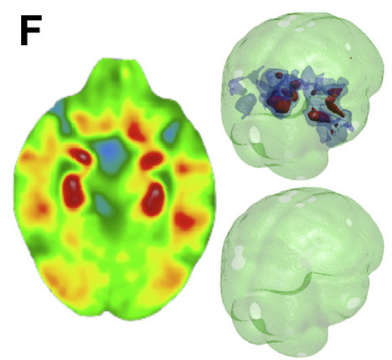

TBM, $P_{c}=1$

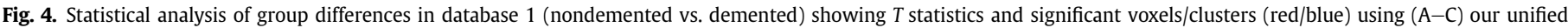

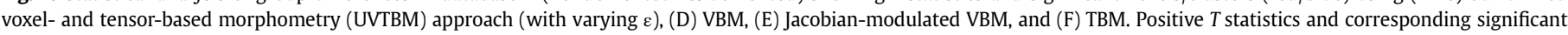

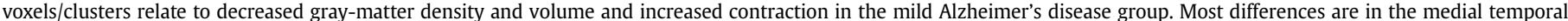

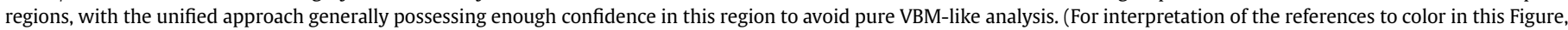
the reader is referred to the web version of this article.)

(Du et al., 2007; Gee et al., 2003). We also compared these UVTBM findings against the traditional VBM, Jacobian-modulated VBM, and TBM analyses, as shown in Fig. 6 for AD and Fig. 7 for FTD. Again, we see that UVTBM achieves consistent results across the error tolerance parameter choices that were explored here $(\varepsilon)$ and reveals a cortical atrophy pattern similar to VBM without modulation. We also see that Jacobian modulation applied in its standard global approach to AD subjects in Fig. 6 E reduces sensitivity to changes in the frontoparietal cortex, where registration accuracy is more variable. This effect is less evident but still observable in the FTD comparison.

\section{Discussion}

In this article, we described a novel unified VBM + TBM framework where the registration confidence is used to weight the

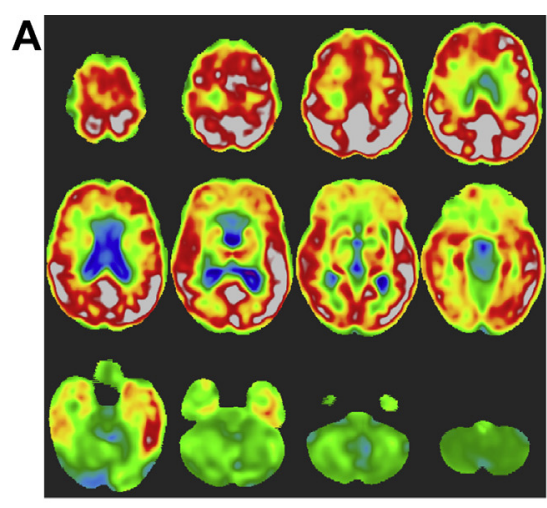

Panel 1
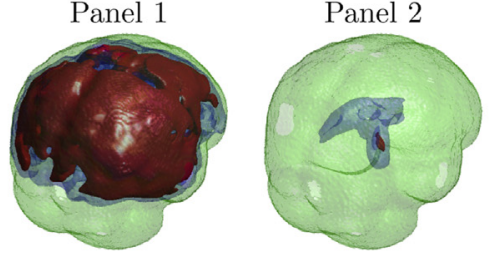

Alzheimer's disease
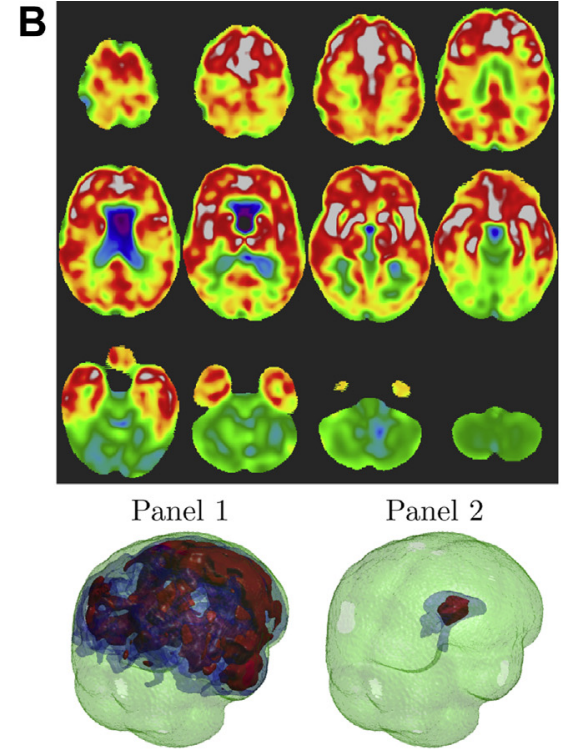

Fronto-temporal dementia
T-stat.

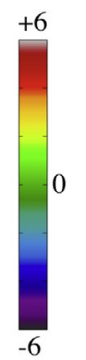

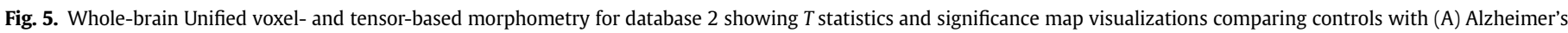

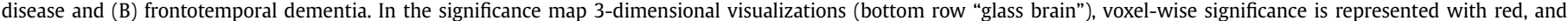

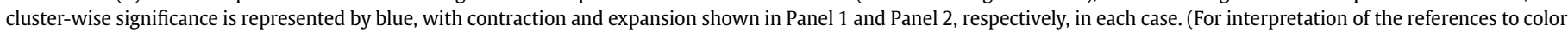
in this Figure, the reader is referred to the web version of this article.) 
A

B

C

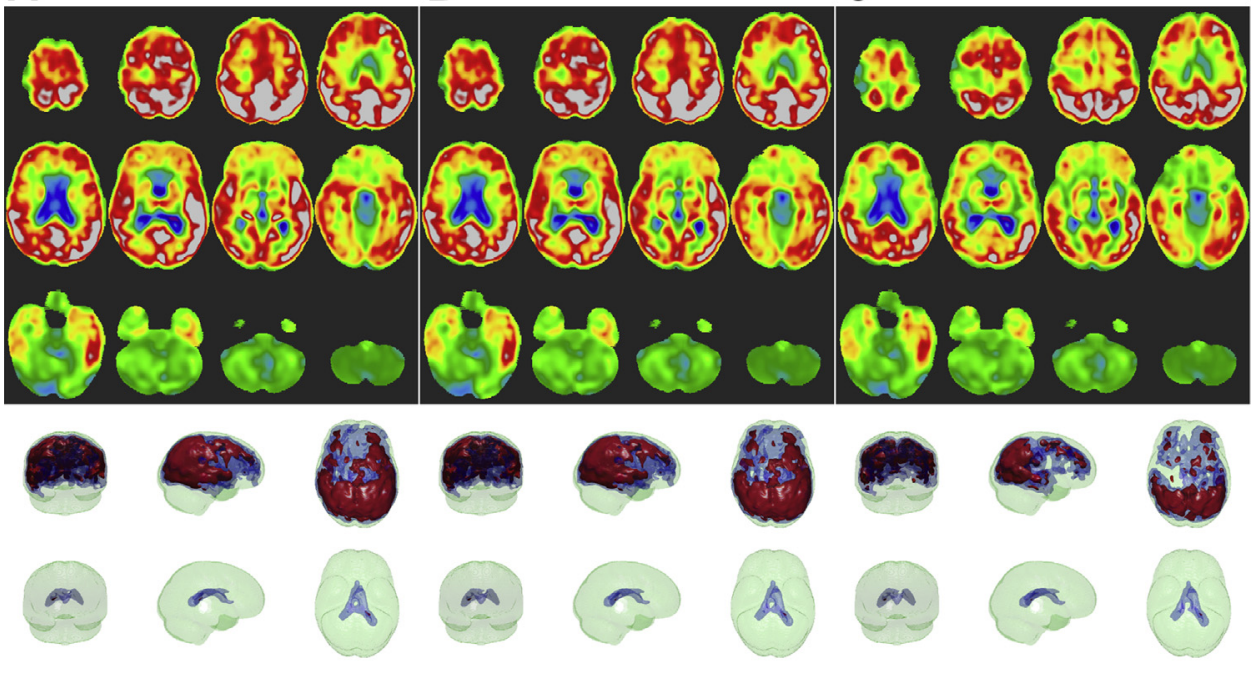

UVTBM, $\epsilon=0.25$

UVTBM, $\epsilon=0.5$

UVTBM, $\epsilon=0.75$

D

$E$

$\mathbf{F}$

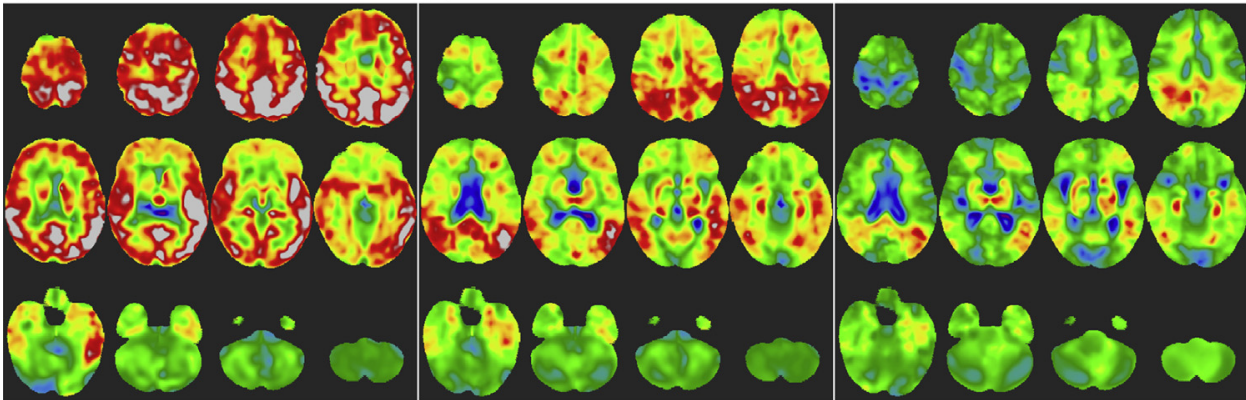

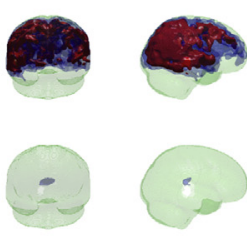

VBM, $P_{c}=0$
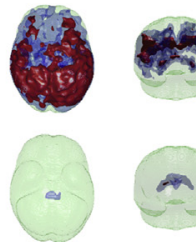

Jac. Mod. VBM, $P_{c}=0.5$
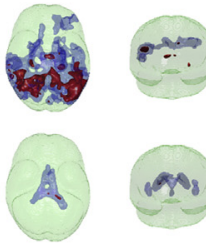

TBM, $P_{c}=1$

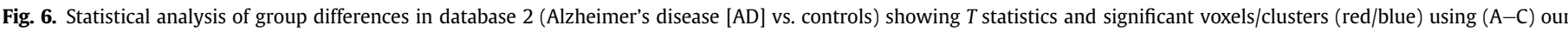

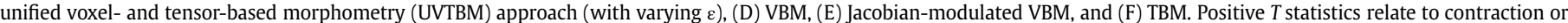

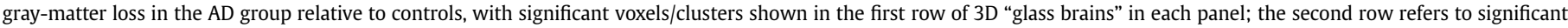

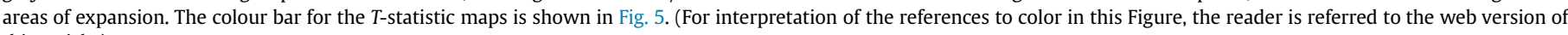
this article.)

relative influence of VBM and TBM in the resulting unified map. The method for estimating registration confidence made use of a surrogate distance-based measure, and other surrogate measures such as regional overlap and local similarity could also be used to a similar effect. These types of surrogates have been employed in multi-atlas label fusion techniques and have thus been shown to effectively estimate registration confidence, with the maps generated here revealing heterogeneous registration accuracy across the brain. These findings agree well with a recent fiducial-based evaluation of registration accuracy (Pereira et al., 2010) that also found greater accuracy in internal regions compared with the external regions of the brain. This heterogeneity affects the interpretation of VBM, modulated VBM, and TBM results, which are traditionally assumed to be objective and unbiased toward specific brain regions. Our unified approach makes use of these confidence estimates to provide a more objective measure that is less sensitive to the regional biases in registration accuracy.
The choice of the tolerance parameter, $\varepsilon$, globally affects the confidence maps and relative weighting of VBM and TBM features in the unified approach. We performed analysis with range of values, and we did not explore methods for optimal choice of this parameter. However, the choice of this parameter is highly dependent on how the residual spatial variability, $\left\|r-\mu_{r}\right\|$, is approximated; thus, the parameter should be tuned according to the approximation method used. Alternatively, if expert-placed fiducials or landmarks were available on a training set, these could be used in future to tune the selection of $\varepsilon$ against gold-standard estimates of registration uncertainty.

This work focussed on 2 related and widely used techniques for morphometry, VBM and TBM; however, another popular technique for assessing tissue atrophy is cortical thickness analysis (Fischl and Dale, 2000). We expect that techniques that employ estimates of regional registration confidence in the analysis of cortical thickness would also lead to greater statistical power; however, we leave this 
A

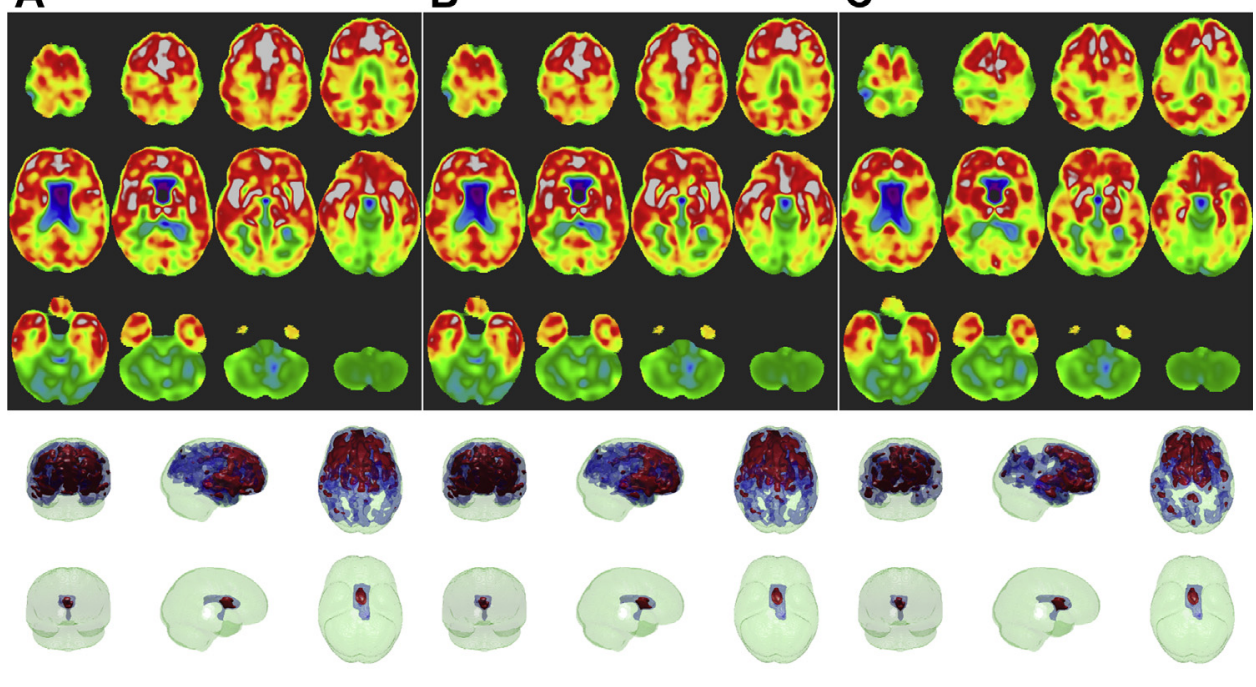

UVTBM, $\epsilon=0.25$

UVTBM, $\epsilon=0.5$

UVTBM, $\epsilon=0.75$

D

E

$\mathbf{F}$

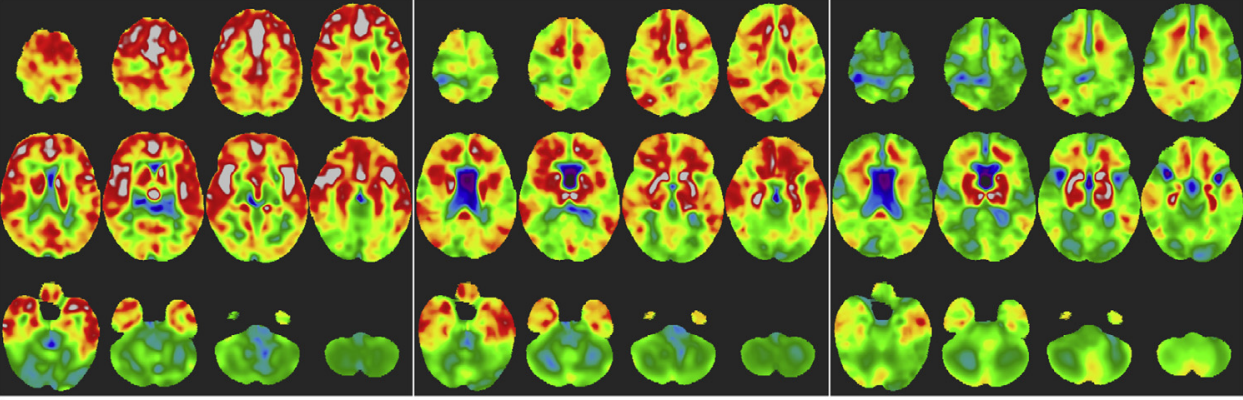

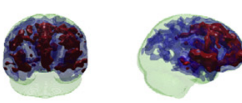

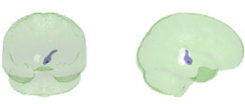

VBM, $P_{c}=0$
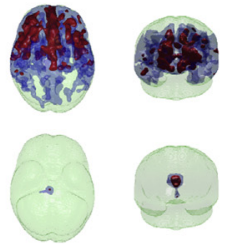

Jac. Mod. VBM, $P_{c}=0.5$
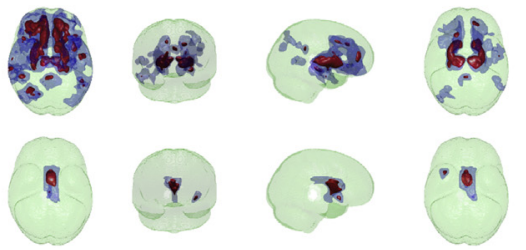

TBM, $P_{c}=1$

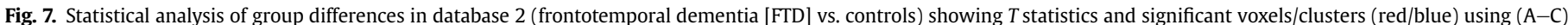

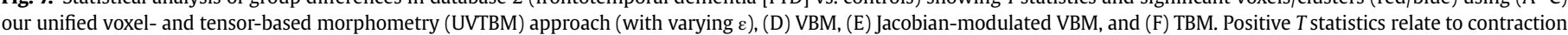

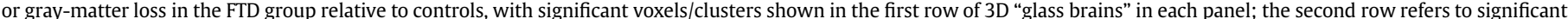

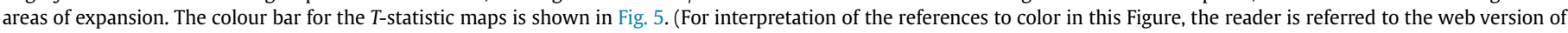
this article.)

investigation to future work. Scalar momentum maps have also been used for morphometric analysis and were applied in a multivariate setting to explore spatial patterns of atrophy with a set of clinical variables (Singh et al., 2010). Our unified VTBM approach would also provide scalar maps and these could be used in the same multivariate partial least-square analysis. Scalar momentum maps more completely describe shape change than TBM and VBM, because they theoretically fully describe the deformation, and comparison of the proposed technique with momentum analysis is also part of our planned future work.

In Figs. 6 and 7, we see that the cortical changes revealed by TBM are more focal, with changes seen in the posterior cingulate and angular gyrus in $\mathrm{AD}$, and medial frontal and anterior cingulate cortices in FTD, whereas VBM revealed more widespread changes. These widespread significant changes are somewhat diluted when the Jacobian is mixed into the VBM maps, although this mixture does capture some subcortical changes near the ventricles that are found using TBM (where the determinant of Jacobian is more likely to be accurate). The proposed VTBM provides a map similar to the Jacobian-modulated VBM, however, with higher sensitivity to the cortical changes that are observed with VBM. The results are similar for the range of epsilon values tested, although more TBM-like behavior is seen with higher epsilon, as was observed in the other examples. We also note that, compared with other TBM studies on similar populations, our implementation of TBM appears to be less sensitive, relative to VBM. This could be because of the amount of regularization used in the registration or because Gaussian smoothing was not applied to the Jacobian determinant maps. We plan to explore the effect of optimal regularization and Gaussian smoothing in future work, with the expectation that optimal application of TBM would lead to improvements in both Jacobian-modulated VBM and our proposed VTBM. Because further optimization of TBM and VBM implementations is possible, we do not suggest that our results show superiority of 
one method over another but instead imply that the locally adaptive combination of TBM and VBM can potentially lead to improved results.

We proposed a novel model for combining VBM and TBM that takes advantage of the merits of each approach and demonstrated its validity and usefulness for morphometric analysis. Our results have shown that alignment accuracy using high-dimensional nonrigid registration can vary throughout the brain, typically with posterior regions showing lower and subcortical regions showing higher accuracy in registration. Experiments presented in this article using our novel locally adaptive approach to generate SPMs suggest that such adaptive approaches offer greater sensitivity and accuracy for detecting group differences than traditional approaches that do not employ local adaptivity.

\section{Disclosure statement}

The authors have no actual or potential conflicts of interest.

\section{Acknowledgements}

We thank Dr Bruce Miller (University of California, San Francisco) for providing the MRI data for FTD, AD, and control subjects. ARK was supported by an Natural Sciences and Engineering Research Council of Canada (NSERC) Canada Graduate Scholarship. We also acknowledge the providers of the Open Access Structural Imaging Studies (grant numbers P50 AG05681, P01 AG03991, R01 AG021910, P20 MH071616, and U24 RR021382).

This work is supported by grant funding from the Natural Sciences and Engineering Research Council of Canada (NSERC), the Canadian Insititutes for Health Research (CIHR), and the Michael Smith Foundation for Health Research (MSFHR).

\section{References}

Ashburner, J., Friston, K.J., 2000. Voxel-based morphometry-the methods. Neuroimage 11 (6 Pt 1), 805-821.

Ashburner, J., Hutton, C., Frackowiak, R., Johnsrude, I., Price, C., Friston, K., 1998. Identifying global anatomical differences: deformation-based morphometry. Hum. Brain Mapp. 6, 348-357.

Avants, B., Epstein, C., Grossman, M., Gee, J., 2008. Symmetric diffeomorphic image registration with cross-correlation: evaluating automated labeling of elderly and neurodegenerative brain. Med. Image Anal. 12, 26-41.

Beg, M.F., Miller, M.I., Trouvé, A., Younes, L., 2005. Computing large deformation metric mappings via geodesic flows of diffeomorphisms. Int. J. Comput. Vis. 61, 139-157.

Christensen, G.E., Rabbitt, R.D., Miller, M.I., 1996. Deformable templates using large deformation kinematics. IEEE Trans. Image Process. 5, 1435-1447.

Dale, A., Fischl, B., Sereno, M., 1999. Cortical surface-based analysis: I. Segmentation and surface reconstruction. Neuroimage 9, 179-194.
Davatzikos, C., Genc, A., Xu, D., Resnick, S.M., 2001. Voxel-based morphometry using the RAVENS maps: methods and validation using simulated longitudinal atrophy. Neuroimage 14, 1361-1369.

Davatzikos, C., Vaillant, M., Resnick, S.M., Prince, J.L., Letovsky, S., Bryan, R.N., 1996. A computerized approach for morphological analysis of the corpus callosum. J. Comput. Assist. Tomogr. 20, 88-97.

Douaud, G., Smith, S., Jenkinson, M., Behrens, T., Johansen-Berg, H., Vickers, J., James, S., Voets, N., Watkins, K., Matthews, P.M., James, A., 2007. Anatomically related grey and white matter abnormalities in adolescent-onset schizophrenia. Brain 130 (Pt 9), 2375-2386.

Du, A.-T., Schuff, N., Kramer, J.H., Rosen, H.J., Gorno-Tempini, M.L., Rankin, K. Miller, B.L., Weiner, M.W., 2007. Different regional patterns of cortical thinning in Alzheimer's disease and frontotemporal dementia. Brain 130 (Pt 4), 1159-1166.

Fischl, B., Dale, A.M., 2000. Measuring the thickness of the human cerebral cortex from magnetic resonance images. Proc. Natl. Acad. Sci. U.S.A. 97, 11050-11055.

Fischl, B., Salat, D., Busa, E., Albert, M., Dieterich, M., Haselgrove, C., van de Kouwe, A., Killiany, R., Kennedy, D., Klaveness, S., 2002. Whole brain segmentation: automated labeling of neuroanatomical structures in the human brain. Neuron 33, 341-355.

Fischl, B., Sereno, M.I., Dale, A.M., 1999. Cortical surface-based analysis: II: inflation, flattening, and a surface-based coordinate system. Neuroimage 9, 195-207.

Fischl, B., van der Kouwe, A., Destrieux, C., Halgren, E., Ségonne, F., Salat, D.H. Busa, E., Seidman, L.J., Goldstein, J., Kennedy, D., Caviness, V., Makris, N., Rosen, B., Dale, A.M., 2004. Automatically parcellating the human cerebral cortex. Cereb. Cortex 14, 11-22.

Gee, J., Ding, L., Xie, Z., Lin, M., DeVita, C., Grossman, M., 2003. Alzheimer's disease and frontotemporal dementia exhibit distinct atrophy-behavior correlates: a computer-assisted imaging study. Acad. Radiol. 10, 1392-1401.

Hub, M., Kessler, M.L., Karger, C.P., 2009. A stochastic approach to estimate the uncertainty involved in B-spline image registration. IEEE Trans. Med. Imaging $28,1708-1716$.

Khan, A. R., 2011. Improving brain registration and segmentation using anatomical guidance [PhD dissertation]. Simon Fraser University, Burnaby, BC, Canada.

Khan, A.R., Wang, L., Beg, M.F., 2013. Multistructure large deformation diffeomorphic brain registration. IEEE Trans. Biomed. Eng. 60, 544-553.

Klein, A., Andersson, J., Ardekani, B.A., Ashburner, J., Avants, B., Chiang, M.-C. Christensen, G.E., Collins, D.L., Gee, J., Hellier, P., Song, J.H., Jenkinson, M., Lepage, C., Rueckert, D., Thompson, P., Vercauteren, T., Woods, R.P., Mann, J.J., Parsey, R.V., 2009. Evaluation of 14 nonlinear deformation algorithms applied to human brain MRI registration. Neuroimage 46, 786-802.

Kybic, J., 2010. Bootstrap resampling for image registration uncertainty estimation without ground truth. IEEE Trans. Image Process. 19, 64-73.

Marcus, D.S., Fotenos, A.F., Csernansky, J.G., Morris, J.C., Buckner, R.L., 2010. Open Access Series of Imaging Studies: longitudinal MRI data in nondemented and demented older adults. J. Cogn. Neurosci. 22, 2677-2684.

Mechelli, A., Price, C.J., Friston, K.J., Ashburner, J., 2005. Voxel-based morphometry of the human brain: methods and applications. Curr. Med. Imaging Rev. 1, 1-9.

Pereira, J. Xiong, L. Acosta-Cabronero, J. Pengas, G. Williams, G., Nestor, P., 2010. Registration accuracy for VBM studies varies according to region and degenerative disease grouping. Neuroimage 49, 2205-2215.

Risholm, P., Janoos, F., Norton, I., Golby, A.J., Wells, W.M., 2013. Bayesian characterization of uncertainty in intra-subject non-rigid registration. Med. Image Anal. 17, 538-555.

Simpson, I.J., Schnabel, J.A., Groves, A.R., Andersson, J.L., Woolrich, M.W., 2012. Probabilistic inference of regularisation in non-rigid registration. Neuroimage 59, 2438-2451.

Singh, N., Fletcher, P.T., Preston, J.S., Ha, L., King, R., Marron, J.S., Wiener, M., Joshi, S. 2010. Multivariate statistical analysis of deformation momenta relating anatomical shape to neuropsychological measures. Med. Image Comput. Comput. Assist. Interv. 13 (Pt 3), 529-537.

Thacker, N., 2005. Tutorial: A Critical Analysis of Voxel Based Morphometry (VBM). http://www.tina-vision.net/docs/memos/2003-011.pdf. University of Manchester. 3-14-2008. 